\title{
Direct percutaneous endoscopic jejunostomy for jejunal feeding
}

\author{
J. S. Shome, H. Gupta, R. Nicholson and P. A. O'Toole \\ Aintree Centre for Gastroenterology, University Hospital Aintree, Liverpool, UK
}

The aim was to review our experience of direct percutaneous endoscopic jejunostomy (DPEJ) tube placement at Aintree Centre for Gastroenterology, University Hospital Aintree, Liverpool (UHA).

Patients were identified from the percuteneous endoscopic gastrostomy (PEG)/PEJ database maintained at UHA. 20 attempted DPEJ procedures were identified, spanning the time period from December 1997 to October 2005. A retrospective review of all relevant casenotes, clinic follow-up letters, and endoscopy reports was performed. Details were recorded relating to: technical success rates, indications, reasons for post-pyloric feeding, previous procedures attempted prior to DPEJ, technical details of the insertion, complications, details of any re-interventions and clinical outcomes.

DPEJ insertion was attempted in eighteen patients. Two patients underwent two procedures. The indications for artificial enteral nutrition were: upper gastrointestinal malignancy (nine), head and neck cancer (two), Cerebrovascular Accidents (CVA) (two), degenerative neurological conditions (four) and scleroderma (one). Seven patients had previous gastric resections, eleven had intact stomachs. DPEJ was successfully performed in eighteen of twenty attempts (technical success rate: 90\%). Of the two failures, one patient had a successful DPEJ placement at the second attempt. A prior procedure had been attempted (e.g. PEG) in nine of the eighteen patients referred, There were no immediate complications. One patient, who had suffered a large CVA, died from pneumonia the day after DPEJ placement. Only one severe late complication was observed (5.3\%). This was a jejunal volvulus requiring small bowel resection. Moderate complications occurred in four patients (22\%) including: peri-tubal leakage requiring tube replacement (two), PEJ site infection requiring intravenous antibiotics (one) and intermittent bowel obstruction attributed to volvulus (one). Two patients with neurological dysphagia had a further episode of aspiration pneumonia following DPEJ placement. Minor complications occurred in eleven patients including persistent abdominal pain, excoriation at PEJ site, mild tube-leakage and PEJ-related diarrhoea. Re-intervention was required in three patients (17\%). Duration of DPEJ utilization ranged from 0.5-40 months (mean about 9 months, median 8 months). Two patients died within $30 \mathrm{~d}$ of the procedure (30-d mortality: $11 \%)$.

DPEJ can be performed with high success rates and a low rate of complications. This confirms its place as an alternative means of longterm enteral nutrition in patients for whom PEG is contraindicated or impossible. Complication rates were similar to those described in other series and the re-intervention rate was lower than commonly reported for PEG jejunal extensions. 